\title{
Plant polyphenols as dietary antioxidants in human health and disease
}

\author{
Kanti Bhooshan Pandey and Syed Ibrahim Rizvi* \\ Department of Biochemistry; University of Allahabad; Allahabad, India
}

Key words: polyphenols, antioxidants, bioavailability, human diseases

Polyphenols are secondary metabolites of plants and are generally involved in defense against ultraviolet radiation or aggression by pathogens. In the last decade, there has been much interest in the potential health benefits of dietary plant polyphenols as antioxidant. Epidemiological studies and associated meta-analyses strongly suggest that long term consumption of diets rich in plant polyphenols offer protection against development of cancers, cardiovascular diseases, diabetes, osteoporosis and neurodegenerative diseases. Here we present knowledge about the biological effects of plant polyphenols in the context of relevance to human health.

\section{Introduction}

Polyphenols are naturally occurring compounds found largely in the fruits, vegetables, cereals and beverages. Fruits like grapes, apple, pear, cherries and berries contains up to $200-300 \mathrm{mg}$ polyphenols per 100 grams fresh weight. The products manufactured from these fruits, also contain polyphenols in significant amounts. Typically a glass of red wine or a cup of tea or coffee contains about $100 \mathrm{mg}$ polyphenols. Cereals, dry legumes and chocolate also contribute to the polyphenolic intake. ${ }^{1,2}$

Polyphenols are secondary metabolites of plants and are generally involved in defense against ultraviolet radiation or aggression by pathogens. ${ }^{3}$ In food, polyphenols may contribute to the bitterness, astringency, color, flavor, odor and oxidative stability. Towards the end of $20^{\text {th }}$ century, epidemiological studies and associated meta-analyses strongly suggested that long term consumption of diets rich in plant polyphenols offered some protection against development of cancers, cardiovascular diseases, diabetes, osteoporosis and neurodegenerative diseases ${ }^{4,5}$ (Fig. 1). Polyphenols and other food phenolics are the subject of increasing scientific interest because of their possible beneficial effects on human health. This review focuses on the present understanding of the biological effects of dietary polyphenols and their importance in human health and disease.

*Correspondence to: Syed Ibrahim Rizvi; Email: sirizvi@gmail.com Submitted: 07/06/09; Revised: 07/12/09; Accepted: 07//3/09

Previously published online:

www.landesbioscience.com/journals/oximed/article/9498

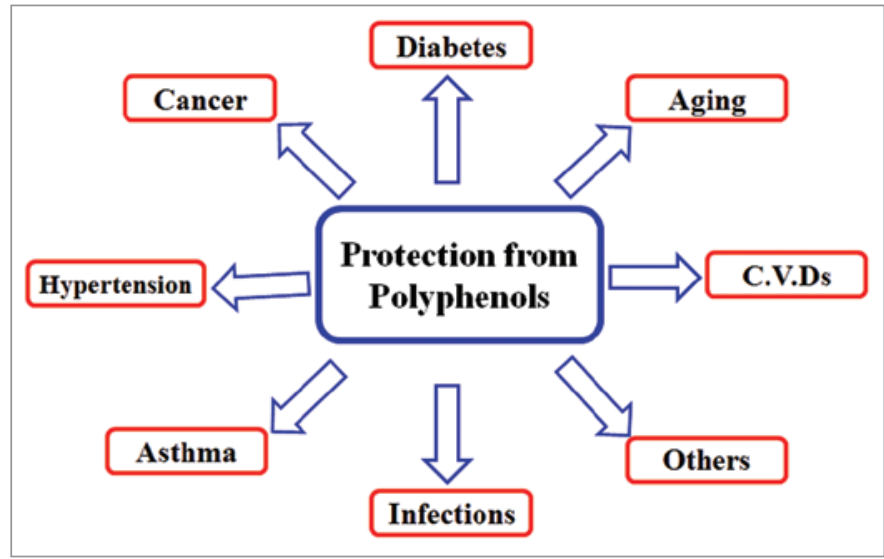

Figure I. Pleiotropic health beneficial effects of dietary plant polyphenols: Polyphenols are naturally occurring compounds found largely in the fruits, vegetables, cereals and beverages. These molecules are secondary metabolites of plants and are generally involved in defense against ultraviolet radiation or aggression by pathogens and may also contribute to the bitterness, astringency of the food. Researchers have explored that these molecules are very good antioxidants and may neutralize the destructive reactivity of undesired reactive oxygen/nitrogen species produced as byproduct during metabolic processes in the body. Epidemiological studies have revealed that polyphenols provide a significant protection against development of several chronic diseases such as cardiovascular diseases (CVDs), cancer, diabetes, infections, aging, asthma etc.

\section{Structure and Classes of Polyphenols}

More than 8,000 polyphenolic compounds have been identified in various plant species. All plant phenolic compounds arise from a common intermediate, phenylalanine, or a close precursor, shikimic acid. Primarily they occur in conjugated forms, with one or more sugar residues linked to hydroxyl groups, although direct linkages of the sugar (polysaccharide or monosaccharide) to an aromatic carbon also exist. Association with other compounds, like carboxylic and organic acids, amines, lipids and linkage with other phenol is also common. ${ }^{6}$ Polyphenols may be classified into different groups as a function of the number of phenol rings that they contain and on the basis of structural elements that bind these rings to one another. The main classes include phenolic acids, flavonoids, stilbenes and lignans. ${ }^{2}$ Figure 2 illustrates the different groups of polyphenols and their chemical structures. 


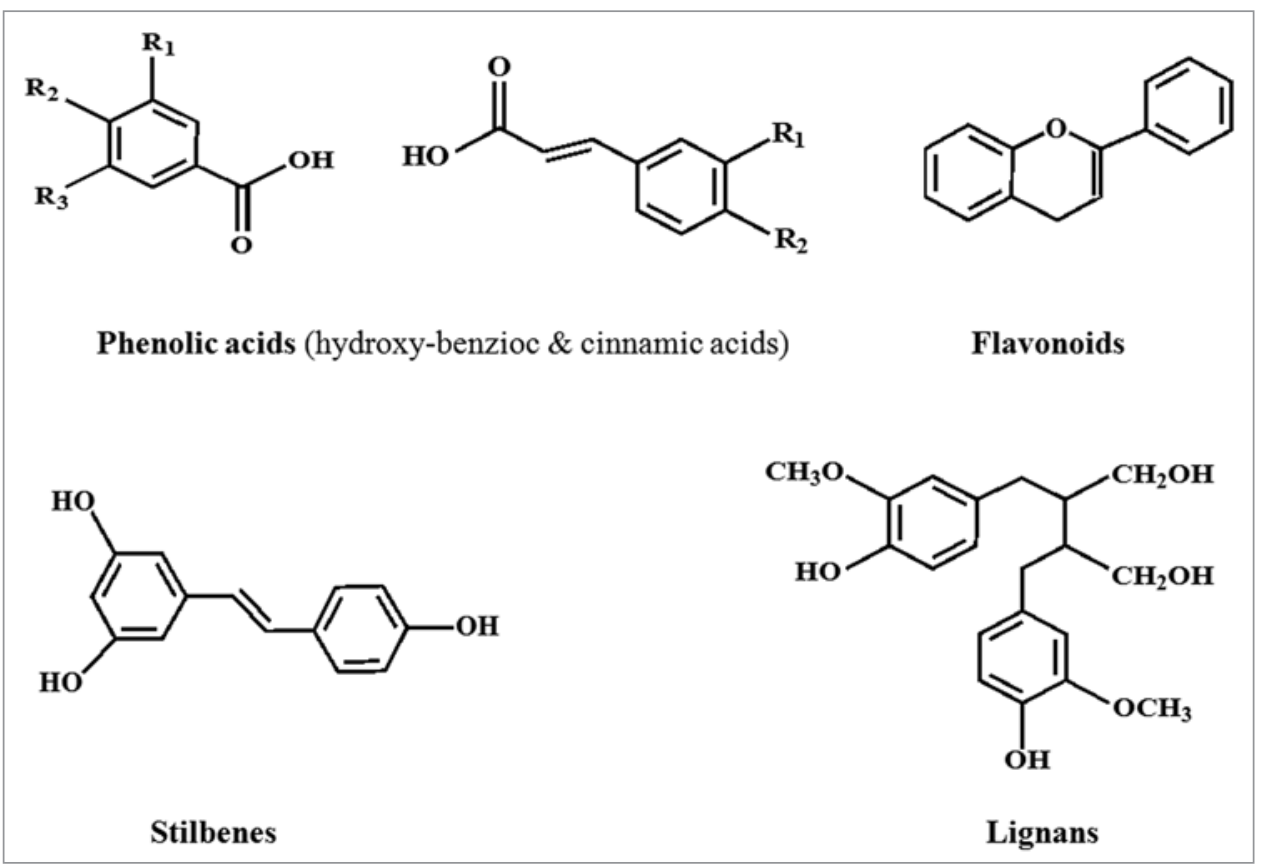

Figure 2. Chemical structures of the different classes of polyphenols. Polyphenols are classified on the basis of the number of phenol rings that they contain and of the structural elements that bind these rings to one another. They are broadly dived in four classes; Phenolic acids, flavonoids, stilbenes and lignans. Phenolic acids are further divided into hydroxyl benzoic and hydroxyl cinnamic acids. Phenolic acids account for about a third of the polyphenolic compounds in our diet and are found in all plant material, but are particularly abundant in acidic-tasting fruits. Caffeic acid, gallic acid, ferulic acid are some common phenolic acids. Flavonoids are most abundant polyphenols in human diet and share a common basic structure consist of two aromatic rings, which are bound together by three carbon atoms that form an oxygenated heterocycle. Biogenetically, one ring usually arises from a molecule of resorcinol, and other ring is derived from the shikimate pathway. Stilbenes contain two phenyl moieties connected by a twocarbon methylene bridge. Most stilbenes in plants act as antifungal phytoalexins, compounds that are synthesized only in response to infection or injury. The most extensively studied stilbene is resveratrol. Lignans are diphenolic compounds that contain a 2,3-dibenzylbutane structure that is formed by the dimerization of two cinnamic acid residues.

\section{Phenolic Acids}

Phenolic acids are found abundantly in foods and divided into two classes: derivatives of benzoic acid and derivatives of cinnamic acid. The hydroxybenzoic acid content of edible plants is generally low, with the exception of certain red fruits, black radish and onions, which can have concentrations of several tens of milligrams per kilogram fresh weight. ${ }^{7}$ The hydroxycinnamic acids are more common than hydroxybenzoic acids and consist chiefly of $p$-coumaric, caffeic, ferulic and sinapic acids.

\section{Flavonoids}

Favonoids comprise the most studied group of polyphenols. This group has a common basic structure consisting of two aromatic rings bound together by three carbon atoms that form an oxygenated heterocycle (Fig. 2). More than 4,000 varieties of flavonoids have been identified, many of which are responsible for the attractive colours of the flowers, fruits and leaves. ${ }^{8}$ Based on the variation in the type of heterocycle involved, flavonoids may be divided into six subclasses: flavonols, flavones, flavanones, flavanols, anthocyanins and isoflavones (Fig. 3). Individual differences within each group arise from the variation in number and arrangement of the hydroxyl groups and their extent of alkylation and/or glycosylation. ${ }^{2}$ Quercetin, myricetin, catechins etc., are some most common flavonoids.

\section{Stilbenes}

Stilbenes contain two phenyl moieties connected by a two-carbon methylene bridge. Occurrence of stilbenes in the human diet is quite low. Most stilbenes in plants act as antifungal phytoalexins, compounds that are synthesized only in response to infection or injury. One of the best studied, naturally occurring polyphenol stilbene is resveratrol $\left(3,4^{\prime}, 5\right.$-trihydroxystilbene), found largely in grapes. A product of grapes, red wine also contains significant amount of resveratrol.

\section{Lignans}

Lignans are diphenolic compounds that contain a 2,3-dibenzylbutane structure that is formed by the dimerization of two cinnamic acid residues (Fig. 2). Several lignans, such as secoisolariciresinol, are considered to be phytoestrogens. The richest dietary source is linseed, which contains secoisolariciresinol (up to $3.7 \mathrm{~g} / \mathrm{kg}$ dry weight) and low quantities of matairesinol.'

\section{Occurrence and Content}

Distribution of phenolics in plants at the tissue, cellular and sub cellular levels is not uniform. Insoluble phenolics are found in cell walls, while soluble phenolics are present within the plant cell vacuoles. ${ }^{10}$ Certain polyphenols like quercetin are found in all plant products; fruit, vegetables, cereals, fruit juices, tea, wine, infusions etc., whereas flavanones and isoflavones are specific to particular foods. In most cases, foods contain complex mixtures of polyphenols. The outer layers of plants contain higher levels of phenolics than those located in their inner parts. ${ }^{11}$ Numerous factors affect the polyphenol content of plants, these include degree of ripeness at the time of harvest, environmental factors, processing and storage. Polyphenolic content of the foods are greatly affected by environmental factors as well as edaphic factors like soil type, sun exposure, rainfall etc. The degree of ripeness considerably affects the concentrations and proportions of various 
polyphenols. ${ }^{12}$ In general, it has been observed that phenolic acid content decreases during ripening, whereas anthocyanin concentrations increase. Many polyphenols, especially phenolic acids, are directly involved in the response of plants to different types of stress: they contribute to healing by lignifications of damaged areas possess antimicrobial properties, and their concentrations may increase after infection. ${ }^{13}$

Another factor that directly affects the polyphenol content of the foods is storage. Studies have proved that polyphenolic content of the foods change on storage, the reason is easy oxidation of these polyphenols. ${ }^{12}$ Oxidation reactions result in the formation of more or less polymerized substances, which lead to changes in the quality of foods, particularly in color and organoleptic characteristics. Such changes may be beneficial, as is the case with black tea or harmful as in browning of fruit. Storage of wheat flour results in marked loss of phenolic acids. ${ }^{14}$ After six months of storage, flour contained the same phenolic acids in qualitative terms, but their concentrations were $70 \%$ lower compared with fresh. Cold storage, in contrast, has slight effect on the content of polyphenols in apples, pears or onions..$^{15}$ Cooking also has a major effect on concentration of polyphenols. Onions and tomatoes lose between $75 \%$ and $80 \%$ of their initial quercetin content after boiling for $15 \mathrm{~min}, 65 \%$ after cooking in a microwave oven, and 30\% after frying. ${ }^{16}$

\section{Bioavailability of Polyphenols}

Bioavailability is the proportion of the nutrient that is digested, absorbed and metabolized through normal pathways. Bioavailability of each and every polyphenol differs however there is no relation between the quantity of polyphenols in food and their bioavailability in human body. Generally, aglycones can be absorbed from the small intestine; however most polyphenols are present in food in the form of esters, glycosides or polymers that cannot be absorbed in native form. ${ }^{17}$ Before absorption, these compounds must be hydrolyzed by intestinal enzymes or by colonic microflora. During the course of the absorption, polyphenols undergo extensive modification; in fact they are conjugated in the intestinal cells and later in the liver by methylation, sulfation and/or glucuronidation. ${ }^{18}$ As a consequence, the forms reaching the blood and tissues are different from those present in food and<smiles>[Y9]c1cc(C2CC(=O)c3c(O)cc(O)cc3O2)cc([Y])c1[R16]</smiles>

Flavanones
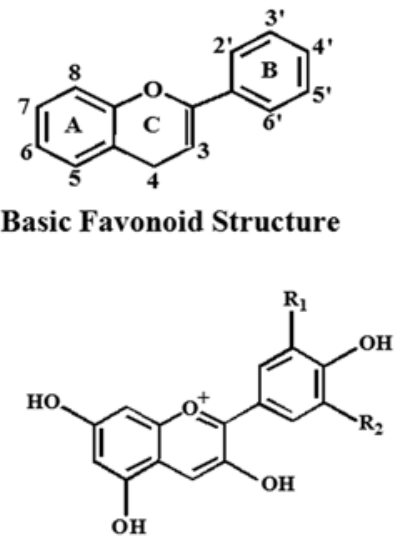

Anthocyanins
Basic Favonoid Structure<smiles>[Y]c1cc(C2Oc3cc(O)cc(O)c3CC2O)cc([R])c1[R1]</smiles>

Flavanols

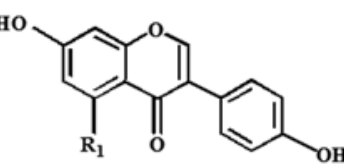

Isoflavones
Figure 3. Chemical structures of sub-classes of flavonoids. Based on the variation in the type of flavones, anthocyanins and isoflavones. Individual differences within each group arise from the variation the hydroxyl groups and their extent of alkylation and/or glycosylavanones (such as naringenin and taxifolin), have an unsaturated carbon-carbon bond in the $\mathrm{C}$ ring. cyanidin), are characterized by the presence of an oxonium ion on the $C$ ring and are highly coloured as a consequence and in isoflavones (such as genistein), the $B$ ring is attached to the $C$ ring in the 3-position, rather than the 2-position as is the case with the other flavonoids.

it is very difficult to identify all the metabolites and to evaluate their biological activity. ${ }^{19}$ Importantly it is the chemical structure of polyphenols and not its concentration that determines the rate and extent of absorption and the nature of the metabolites circulating in the plasma. The most common polyphenols in our diet are not necessarily those showing highest concentration of active metabolites in target tissues; consequently the biological properties of polyphenols greatly differ from one polyphenol to another. Evidence, although indirect, of their absorption through the gut barrier is given by the increase in the antioxidant capacity of the plasma after the consumption of polyphenols-rich foods. ${ }^{20,21}$

Polyphenols also differs in their site of absorption in humans. Some of the polyphenols are well absorbed in the gastro-intestinal tract while others in intestine or other part of the digestive tract. In foods, all flavonoids except flavanols exist in glycosylated forms. The fate of glycosides in the stomach is not clear yet. Most of the glycosides probably resist acid hydrolysis in the stomach and thus arrive intact in the intestine $e^{22}$ where only aglycones and few glucosides can be absorbed. Experimental studies carried out in rats ${ }^{23}$ showed that the absorption at gastric level is possible for some flavonoids, such as quercetin, but not for their 
glycosides. Moreover it has been recently shown that, in rats and mice, anthocyanins are absorbed from the stomach. ${ }^{17,24}$

It was suggested that glucosides could be transported into enterocytes by the sodium dependent glucose transporter SGLT1, and then hydrolyzed by a cytosolic $\beta$-glucosidase. However the effect of glucosylation on absorption is less clear for isoflavones than for quercetin. ${ }^{12}$ Proanthocyanidins differ from most of other plant polyphenols because of their polymeric nature and high molecular weight. This particular feature should limit their absorption through the gut barrier, and oligomers larger than trimers are unlikely to be absorbed in the small intestine in their native forms. ${ }^{17,25}$

It was observed that the hydroxycinnamic acids, when ingested in the free form, are rapidly absorbed by the small intestine and are conjugated as the flavonoids. ${ }^{26}$ However these compounds are naturally esterified in plant products and esterification impairs their absorption because intestinal mucosa, liver and plasma do not possess esterases capable of hydrolyzing chlorogenic acid to release caffeic acid, and hydrolysis can be performed only by the microflora present in colon. ${ }^{27}$ Though most of the poyphenols get absorbed in gastrointestinal tract and intestine but there are some poyphenols which are not absorbed in these locations. These polyphenols reach the colon, where microflora hydrolyze glycosides into aglycones and extensively metabolize these aglycones into various aromatic acids. ${ }^{28}$

Aglycones are split by the opening of the heterocycle at different points depending on their chemical structure, and thus produce different acids that are further metabolized to derivatives of benzoic acid. After absorption, polyphenols go to several conguation processes. These processes mainly include methylation, sulfation and glucuronidation, representing a metabolic detoxication process, common to many xenobiotics, that facilitates their biliary and urinary elimination by increasing their hydrophilicity. The methylation of poyphenols is also quite specific it generally occurs in the C3-position of the polyphenol, but it could occur in the C4'-position: in fact a notable amount of 4'-methylepigallocatechin has been detected in human plasma after tea ingestion. ${ }^{29}$ Enzymes like sulfo-transferases catalyze the transfer of a sulfate moiety during process of sulphonation. The sulfation occurs mainly in the liver, but the position of sulfation for polyphenols have not been clearly identified yet. ${ }^{30}$ Glucuronidation occurs in the intestine and in the liver, and the highest rate of conjugation is observed in the C3-position. ${ }^{31}$ The conjugation mechanisms are highly efficient and free aglycones are generally either absent, or present in low concentrations in plasma after consumption of nutritional doses; an exception are green tea catechins, whose aglycones can constitute a significant proportion of the total amount in plasma. ${ }^{32}$

It is important to identify the circulating metabolites, including the nature and the positions of the conjugating groups on the polyphenol structure, because the positions can affect the biological properties of the conjugates. Polyphenol metabolites circulate in the blood bound to proteins; in particular albumin represents the primary protein responsible for the binding. Albumin plays an important role in bioavailability of polyphenols. The affinity of polyphenols for albumin varies according to their chemical structure. ${ }^{33}$
Binding to albumin may have consequences for the rate of clearance of metabolites and for their delivery to cells and tissues. It is possible that the cellular uptake of metabolites is proportional to their unbound concentration. Finally, it is still unclear if the polyphenols have to be in the free form to exert their biological activity, or the albumin-bound polyphenols can exert some biological activity. ${ }^{17,34}$

Accumulation of polyphenols in the tissues is the most important phase of polyphenol metabolism because this is the concentration which is biologically active for exerting the effects of polyphenols. Studies have shown that the polyphenols are able to penetrate tissues, particularly those in which they are metabolized such as intestine and liver. Excretion of polyphenols with their derivatives occurs through urine and bile. It has been observed that the extensively conjugated metabolites are more likely to be eliminated in bile, whereas small conjugates, such as monosulfates, are preferentially excreted in urine. Amount of metabolites excreted in urine is roughly correlated with maximum plasma concentrations. Urinary excretion percentage is quite high for flavanones from citrus fruit and decreases from isoflavones to flavonols. Thus the health beneficial effects of the polyphenols depend upon both the intake and bioavailability. ${ }^{17}$

\section{Polyphenols and Human Diseases}

Epidemiological studies have repeatedly shown an inverse association between the risk of chronic human diseases and the consumption of polyphenolic rich diet. ${ }^{1,5}$ The phenolic groups in polyphenols can accept an electron to form relatively stable phenoxyl radicals, thereby disrupting chain oxidation reactions in cellular components. ${ }^{26}$ It is well established that polyphenol-rich foods and beverages may increase plasma antioxidant capacity. This increase in the antioxidative capacity of plasma following the consumption of polyphenol-rich food may be explained either by the presence of reducing polyphenols and their metabolites in plasma, by their effects upon concentrations of other reducing agents (sparing effects of polyphenols on other endogenous antioxidants), or by their effect on the absorption of pro-oxidative food components, such as iron. ${ }^{1}$ Consumption of antioxidants has been associated with reduced levels of oxidative damage to lymphocytic DNA. Similar observations have been made with plyphenol-rich food and beverages indicating the protective effects of polyphenols. ${ }^{35}$ There are increasing evidences that as antioxidants, polyphenols may protect cell constituents against oxidative damage and, therefore, limit the risk of various degenerative diseases associated with oxidative stress. ${ }^{36-38}$

\section{Cardio-Protective Effect}

Number of studies has demonstrated that consumption of polyphenols limits the incidence of coronary heart diseases. ${ }^{39-41}$ Atherosclerosis is a chronic inflammatory disease that develops in lesion-prone regions of medium-sized arteries. Atherosclerotic lesions may be present and clinically silent for decades before becoming active and producing pathological conditions such as acute myocardial infarction, unstable angina or sudden cardiac death. ${ }^{42}$ Polyphenols are potent inhibitors of LDL oxidation and 
this type of oxidation is considered to be a key mechanism in development of atherosclerosis. ${ }^{43}$ Other mechanisms by which polyphenols may be protective against cardiovascular diseases are antioxidant, anti-platelet, anti-inflammatory effects as well as increasing HDL, and improving endothelial function. ${ }^{44}$ Polyphenols may also contribute to stabilization of the atheroma plaque.

Quercetin, the abundant polyphenol in onion has been shown to be inversely associated with mortality from coronary heart disease by inhibiting the expression of metalloproteinase 1 (MMP1), and the disruption of atherosclerotic plaques. ${ }^{44}$ Tea catechins have been shown to inhibit the invasion and proliferation of the smooth muscle cells in the arterial wall, a mechanism that may contribute to slow down the formation of the atheromatous lesion. ${ }^{45}$ Polyphenols may also exert antithrombotic effects by means of inhibiting platelet aggregation. Consumption of red wine or non-alcoholic wine reduces bleeding time and platelet aggregation. Thrombosis induced by stenosis of coronary artery is inhibited when red wine or grape juice is administrated. ${ }^{46}$ Polyphenols can improve endothelial dysfunction associated with different risk factors for atherosclerosis before the formation of plaque; its use as a prognostic tool for coronary heart diseases has also been proposed. ${ }^{47}$ It has been observed that consumption of black tea about $450 \mathrm{ml}$ increases artery dilation 2 hours after intake and consumption of $240 \mathrm{~mL}$ red wine for 30 days countered the endothelial dysfunction induced by a high fat diet. ${ }^{48}$ Long term regular intake of black tea was found to lower blood pressure in a cross-sectional study of 218 women above 70 years of age. Excretion of 4-O-methylgallic acid (4OMGA, a biomarker for tea polyphenols in body) was monitored. A higher consumption of tea and therefore higher excretion of 4OMGA were associated with lower blood pressure (BP). Tea polyphenols may be the components responsible for the lowering of BP. The effect may be due to antioxidant activity as well as improvement of endothelial function or estrogen like activity. ${ }^{44}$

Resveratrol, the wine polyphenol prevents the platelet aggregation via preferential inhibition of cyclooxygenase 1(COX 1) activity, which synthesizes thromboxane A2, an inducer of the platelet aggregation and vasoconstrictor. ${ }^{49}$ In addition to this, resveratrol is capable of relaxing the isolated arteries and rat aortic rings. The ability to stimulate $\mathrm{Ca}^{++}$ activated $\mathrm{K}^{+}$channels and to enhance nitric oxide signaling in the endothelium are other pathways by which resveratrol exerts vasorelaxant activity. ${ }^{49,50}$ Direct relation between cardiovascular diseases (CVDs) and oxidation of LDL is now well established. Oxidation of LDL particles is strongly associated with the risk of coronary heart diseases and myocardial infarctions. Studies have shown that resveratrol potentially inhibits the oxidation of the LDL particles via chelating copper or by direct scavenging of the free radicals. Resveratrol is the active compound in red wine which is attributed for "French Paradox", the low incidence of CVD despite the intake of high-fat diet and smoking among French. ${ }^{51,52}$ Association between polyphenol intake or the consumption of polyphenol-rich foods and incident of cardiovascular diseases were also examined in several epidemiological studies and it was found that consumption of polyphenol rich diet have been associated to a lower risk of myocardial infarction in both case-control and cohort studies. ${ }^{53}$

\section{Anti-Cancer Effect}

Effect of polyphenols on human cancer cell lines, is most often protective and induce a reduction of the number of tumors or of their growth. ${ }^{54}$ These effects have been observed at various sites, including mouth, stomach, duodenum, colon, liver, lung, mammary gland or skin. Many polyphenols, such as quercetin, catechins, isoflavones, lignans, flavanones, ellagic acid, red wine polyphenols, resveratrol and curcumin have been tested; all of them showed protective effects in some models although their mechanisms of action were found to be different. ${ }^{55}$

Development of cancer or carcinogenesis is a multistage and microevolutionary process. Into the three major stages of carcinogenesis: initiation, promotion and progression. Initiation is a heritable aberration of a cell. Cells so initiated can undergo transformation to malignancy if promotion and progression follow. Promotion, on the other hand, is affected by factors that do not alter DNA sequences and involves the selection and clonal expansion of initiated cells.

Several mechanisms of action have been identified for chemoprevention effect of polyphenols, these include estrogenic/antiestrogenic activity, antiproliferation, induction of cell cycle arrest or apoptosis, prevention of oxidation, induction of detoxification enzymes, regulation of the host immune system, anti-inflammatory activity and changes in cellular signaling. ${ }^{44}$

Polyphenols influence the metabolism of pro-carcinogens by modulating the expression of cytochrome P450 enzymes involved in their activation to carcinogens. They may also facilitate their excretion by increasing the expression of phase II conjugating enzymes. This induction of phase II enzymes may have its origin in the toxicity of polyphenols. ${ }^{1}$ Polyphenols can form potentially toxic quinones in the body that are, themselves, substrates of these enzymes. The intake of polyphenols could then activate these enzymes for their own detoxication and, thus, induce a general boosting of our defenses against toxic xenobiotics. ${ }^{56}$ It has been demonstrated that tea catechins in the form of capsules when given to men with high-grade prostate intraepithelial neoplasia (PIN) demonstrated cancer preventive activity by inhibiting the conversion of high grade PIN lesions to cancer. ${ }^{57}$

Theaflavins and thearubigins, the abundant polyphenols in black tea have also been shown to possess strong anticancer property. Black tea polyphenols were found to inhibit proliferation and increase apoptosis in $\mathrm{Du} 145$ prostate carcinoma cells. Higher level of insulin like growth factor-1 (IGF-1) was found to be associated with a higher risk of development of prostate cancer. IGF-1 binding to its receptor is a part of signal transduction pathway which causes cell proliferation. ${ }^{58}$ Black tea polyphenol addition was found to block IGF-1 induced progression of cells into $S$ phase of cell cycle at a dose of $40 \mathrm{mg} / \mathrm{ml}$ in prostate carcinoma cells..$^{58}$

Quercetin has also been reported to possess anticancer property against benzo(a)pyrene induced lung carcinogenesis in mice, an effect attrtibuted to its free radical scavenging activity. ${ }^{59}$ 
Resveratrol prevents all stages of development of cancer and has been found to be effective in most types of cancer including lung, skin, breast, prostate, gastric and colorectal cancer. It has also been shown to suppress angiogenesis and metastasis. Extensive data in human cell cultures indicate that resveratrol can modulate multiple pathways involved in cell growth, apoptosis and inflammation. The anti-carcinogenic effects of resveratrol appears to be closely associated with its antioxidant activity, and it has been shown to inhibit cyclooxygenase, hydroperoxidase, protein kinase C, Bcl-2 phosphorylation, Akt, focal adhesion kinase, NFKB, matrix metalloprotease- 9 and cell cycle regulators. ${ }^{60}$ These and other in vitro and in vivo studies provide a rationale in support of the use of dietary polyphenols in human cancer chemoprevention, in a combinatorial approach with either chemotherapeutic drugs or cytotoxic factors for efficient treatment of drug refractory tumor cells.

\section{Anti-Diabetic Effect}

Impairment in glucose metabolism leads to physiological imbalance with the onset of the hyperglycemia and subsequently diabetes mellitus. There are two main categories of diabetes; type-1 and type-2. Studies have shown that several physiological parameters of the body get altered in the diabetic conditions. ${ }^{61,62}$ Long term effects of diabetes include progressive development of specific complements such as retinopathy, which affects eyes and lead to blindness; nephropathy in which the renal functions are altered or disturbed and neuropathy which is associated with the risks of amputations, foot ulcers and features of autonomic disturbance including sexual dysfunctions. Numerous studies report the antidiabetic effects of polyphenols. Tea catechins have been investigated for their anti-diabetic potential. ${ }^{63,64}$ Polyphenols may affect glycemia through different mechanisms, including the inhibition of glucose absorption in the gut or of its uptake by peripheral tissues. The hypoglycemic effects of diacetylated anthocyanins at a $10 \mathrm{mg} / \mathrm{kg}$ diet dosage were observed with maltose as a glucose source, but not with sucrose or glucose. ${ }^{65}$ This suggests that these effects are due to an inhibition of $\alpha$-glucosidase in the gut mucosa. Inhibition of $\alpha$-amylase and sucrase in rats by catechin at a dose of about $50 \mathrm{mg} / \mathrm{kg}$ diet or higher was also observed.

The inhibition of intestinal glycosidases and glucose transporter by polyphenols has been studied. ${ }^{66}$ Individual polyphenols, such as (+)catechin, (-)epicatechin, (-)epigallocatechin, epicatechin gallate, isoflavones from soyabeans, tannic acid, glycyrrhizin from licorice root, chlorogenic acid and saponins also decrease S-Glut-1 mediated intestinal transport of glucose. Saponins additionally delay the transfer of glucose from stomach to the small intestine. ${ }^{67}$ Resveratrol has also been reported to act as an anti-diabetic agent. Many mechanisms have been proposed to explain the anti-diabetic action of this stilbene, modulation of SIRT1 is one of them which improves whole-body glucose homeostasis and insulin sensitivity in diabetic rats. ${ }^{50,68} \mathrm{It}$ is reported that in cultured LLC-PK1 cells, high glucose induced cytotoxicity and oxidative stress was inhibited by grape seed polyphenols. Resveratrol inhibits diabetes-induced changes in the kidney (diabetic nephropathy) and significantly ameliorates renal dysfunction and oxidative stress in diabetic rats. Treatment with resveratrol also decreased insulin secretion and delayed the onset of insulin resistance. A possible mechanism was thought to be related to the inhibition of $\mathrm{K}+\mathrm{ATP}$ and $\mathrm{K}+\mathrm{V}$ channel in beta cells. ${ }^{69}$

Onion polyphenols, especially quercetin is known to possess strong anti diabetic activity. A recent study shows that quercetin has ability to protect the alterations in diabetic patients during oxidative stress. Quercetin significantly protected the lipid peroxidation and inhibition antioxidant system in diabetics. ${ }^{70}$ Hibiscus sabdariffa extract contains polyphenolic acids, flavonoids, protocatechuic acid and anthocyanins. A study performed by Lee et al. $^{71}$ showed that polyphenols present in the extracts from Hibiscus sabdariffa attenuate diabetic nephropathy including pathology, serum lipid profile and oxidative markers in kidney. Ferulic acid (FA) is another polyphenol very abundant in vegetables and maize bran. Several lines of evidence have shown that FA acts as a potent anti-diabetic agent by acting at many levels. It was demonstrated that FA lowered blood glucose followed by a significantly increased plasma insulin and a negative correlation between blood glucose and plasma insulin. ${ }^{72,73}$

\section{Anti-Aging Effect}

Aging is the accumulation process of diverse detrimental changes in the cells and tissues with advancing age, resulting in an increase in the risks of disease and death. Among many theories purposed for the explaining the mechanism of aging, free radical/oxidative stress theory is one of the most accepted one. ${ }^{74} \mathrm{~A}$ certain amount of oxidative damage takes place even under normal conditions; however, the rate of this damage increases during the aging process as the efficiency of antioxidative and repair mechanisms decrease. ${ }^{75,76}$ Antioxidant capacity of the plasma is related to dietary intake of antioxidants; it has been found that the intake of antioxidant rich diet is effective in reducing the deleterious effects of aging and behavior. Several researches suggest that the combination of antioxidant/anti-inflammatory polyphenolic compounds found in fruits and vegetables may show efficacy as anti-aging compounds. ${ }^{77,78}$ Subset of the flavonoids known as anthocyanins, are particularly abundant in brightly colored fruits such as berry fruits and concord grapes and grape seeds. Anthocyanins are responsible for the colors in fruits, and they have been shown to have potent antioxidant/anti-inflammatory activities, as well as to inhibit lipid peroxidation and the inflammatory mediators cyclo-oxygenase (COX)-1 and $-2 .{ }^{79}$

Fruit and vegetable extracts that have high levels of flavonoids also display high total antioxidant activity such as spinach, strawberries and blueberries. It is reported that the dietary supplementations (for 8 weeks) with spinach, strawberry or blueberry extracts in a control diet were also effective in reversing age-related deficits in brain and behavioral function in aged rats. ${ }^{80}$ A recent study demonstrates that the tea catechins carry strong anti-aging activity and consuming green-tea rich in these catechins, may delay the onset of aging. ${ }^{81}$

Polyphenols are also beneficial in ameliorating the adverse effects of the aging on nervous system or brain. Paramount 
importance for the relevance of food polyphenols in the protection of the aging brain is the ability of these compounds to cross the blood-brain barrier (BBB), which tightly controls the influx in the brain of metabolites and nutrients as well as of drugs. Resveratrol has been found to consistently prolong the life span; its action is linked to an event called caloric restriction or partial food deprivation. ${ }^{50}$

Grape polyphenol, resveratrol is very recent entry as an antiaging agent. It has been shown that the early target of the resveratrol is the sirtuin class of nicotinamide adenine dinucleotide (NAD)-dependent deacetylases. Seven sirtuins have been identified in mammals, of which SIRT-1 is believed to mediate the beneficial effects on health and longevity of both caloric restriction and resveratrol. ${ }^{82}$ Resveratrol increased insulin sensitivity, decreased the expression of IGF-1 and increased AMP-activated protein kinase $(\mathrm{AMPK})$ and peroxisome proliferator-activated receptor-c coactivator 1a (PGC-1a) activity. When examined for the mechanism, it activated forkhead box O (FOXO), which regulates the expression of genes that contribute both to longevity and resistance to various stresses and insulin-like growth factorbinding protein 1 (IGFBP-1). ${ }^{83}$ There are experimental evidences that resveratrol can extend lifespan in the yeast Saccharomyces cerevisiae, the fruit fly Drosophila melanogaster, the nematode worm C. elegans, and seasonal fish Nothobranchius furzeri. ${ }^{52}$ Recently quercetin has also been reported to exert preventive effect against aging. ${ }^{84}$

\section{Neuro-Protective Effects}

Oxidative stress and damage to brain macromolecules is an important process in neurodegenerative diseases. Alzheimer's disease is one of the most common occurring neurodisorder affecting up to 18 million people worldwide. Because polyphenols are highly antioxidative in nature, their consumption may provide protection in neurological diseases. ${ }^{85}$ It was observed that the people drinking three to four glasses of wine per day had $80 \%$ decreased incidence of dementia and Alzheimer's disease compared to those who drank less or did not drink at all. ${ }^{86}$

Resveratrol, abundantly present in wine scavenges $\mathrm{O}_{2}{ }^{-}$and $\mathrm{OH}^{*}$ in vitro, as well as lipid hydroperoxyl free radicals, this efficient antioxidant activity is probably involved in the beneficial effect of the moderate consume of red wine against dementia in the elderly. Resveratrol inhibits nuclear factor $\mathrm{\kappa B}$ signaling and thus gives protection against microglia-dependent $\beta$-amyloid toxicity in a model of Alzheimer's disease and this activity is related with the activation of the SIRT- $1 .^{82}$ It was found that the consumption of fruit and vegetable juices containing high concentrations of polyphenols, at least three times per week, may play an important role in delaying the onset of Alzheimer's disease. ${ }^{87}$ Polyphenols from fruits and vegetables seem to be invaluable potential agents in neuroprotection by virtue of their ability to influence and modulate several cellular processes such as signaling, proliferation, apoptosis, redox balance and differentiation. ${ }^{88}$

Recently Aquilano et al. ${ }^{89}$ reported that administration of polyphenols provide protective effects against Parkinson's disease, a neurological disorder characterized by degeneration of dopaminergic neurons in the substantia nigra zona compacta. Nutritional studies have linked the consumption of green tea to the reduced risk of developing Parkinson's disease. In animal models epigallocatechin gallate (EGCG) has been shown to exert a protective role against the neurotoxin MPTP (N-methyl-4phenyl-1,2,3,6-tetrahydropyridine), an inducer of a Parkinson'slike disease, either by competitively inhibiting the uptake of the drug, due to molecular similarity or by scavenging MPTPmediated radical formation. EGCG may also protect neurons by activating several signaling pathways, involving MAP kinases which are fundamental for cell survival..$^{90}$ The therapeutic role of catechins in Parkinson's disease is also due to their ability to chelate iron. This property contributes to their antioxidant activity by preventing redox-active transition metal from catalyzing free radicals formation. Moreover, the antioxidant function is also related to the induction of the expression of antioxidant and detoxifying enzymes particularly in the brain, which is not sufficiently endowed of a well-organized antioxidant defense system. ${ }^{89}$ Maize bran polyphenol, ferulic acid is also reported to be beneficial in Alzheimer's disease. This effect is due to its antioxidant and anti-inflammatory properties. ${ }^{72}$

\section{Others}

Except above explained pathological events, polyphenols show several other health beneficial effects. Dietary polyphenols exert preventive effects in treatment of asthma. In asthma the airways react by narrowing or obstructing when they become irritated. This makes it difficult for the air to move in and out. This narrowing or obstruction can cause one or a combination of symptoms such as wheezing, coughing, shortness of breath and chest tightness. Epidemiological evidence that polyphenols might protect against obstructive lung disease come from studies that have reported negative associations of apple intake with prevalence and incidence of asthma, and a positive association with lung function..$^{91,92}$ Increased consumption of the soy isoflavone, genistein, was associated with better lung function in asthmatic patients. ${ }^{93}$ Intake of polyphenols is also reported as beneficial in osteoporosis. Supplementation of diet with genistein, daidzein or their glycosides for several weeks prevents the loss of bone mineral density and trabecular volume caused by the ovariectomy. ${ }^{94}$ Polyphenols also protect skin damages induced from sunlight. Study on animals provide evidence that polyphenols present in the tea, when applied orally or topically, ameliorate adverse skin reactions following UV exposure, including skin damage, erythema and lipid peroxidation. ${ }^{95}$

Black tea polyphenols are reported to be helpful in mineral absorption in intestine as well as to possess antiviral activity. Theaflavins present in black tea were found to have anti HIV-1 activity. These polyphenols inhibited the entry of HIV-1 cells into the target cells. HIV-1 entry into the target cell involves fusion of glycoprotein (GP) and envelope of the virus with the cell membrane of the host cells. Haptad repeat units present at $\mathrm{N}$ and $\mathrm{C}$ terminals of GP41 (membrane protein) on the viral envelope, fuse to form the fusion active GP41 core, which is a six-helical bundle. Theaflavins were found to block the formation 
of this six-helix bundle required for entry of the virus into the host. $^{58}$ Theaflavin 3 3' digallate, and theaflavin 3 ' gallate were found to inhibit Severe Acute Respiratory Syndrome (SARS) corona virus. This antiviral activity was due to inhibition of the chymotrypsin like protease (3CL Pro) which is involved in the proteolytic processing during viral multiplication. ${ }^{58}$

\section{Conclusion}

The results of studies outlined in this review provide a current understanding on the biological effects of polyphenols and their relevance to human health. Polyphenols or polyphenol rich diets provide significant protection against the development and progression of many chronic pathological conditions

\section{References}

1. Scalbert A, Manach C, Morand C, Remesy C. Dietary polyphenols and the prevention of diseases. Crit Rev Food Sci Nutr 2005; 45:287-306.

2. Spencer JP, Abd El Mohsen MM, Minihane AM, Mathers JC. Biomarkers of the intake of dietary polyphenols: strengths, limitations and application in nutrition research. Br J Nutr 2008; 99:12-22.

3. Beckman $\mathrm{CH}$. Phenolic-storing cells: keys to programmed cell death and periderm formation in wilt disease resistance and in general defence responses in plants? Physiol. Mol. Plant Pathol 2000; 57:101-10.

4. Graf BA, Milbury PE, Blumberg JB. Flavonols, flavonones,flavanones and human health: Epidemological evidence. J Med Food 2005; 8:28190.

5. Arts ICW, Hollman PCH. Polyphenols and disease risk in epidemiologic studies. Am J Clin Nutr 2005; 81:317-25.

6. Kondratyuk TP, Pezzuto JM. Natural Product Polyphenols of Relevance to Human Health. Pharm Biol 2004; 42:46-63.

7. Shahidi F, Naczk M. Food phenolics, sources, chemistry, effects, applications. Lancaster, PA: Technomic Publishing Co Inc, 1995.

8. de Groot H, Rauen U. Tissue injury by reactive oxygen species and the protective effects of flavonoids. Fundam Clin Pharmacol 1998; 12: 249-55.

9. Adlercreutz H, Mazur W. Phyto-oestrogens and Western diseases. Ann Med 1997; 29:95-120.

10. Wink M. Compartmentation of secondary metabolites and xenobiotics in plant vacuoles, Adv Bot Res 1997; 25:141-69.

11. Simon BF, Perez-Ilzarbe J, Hernandez T, GomezCordoves C, Estrella I. Importance of phenolic compounds for the characterization of fruit juices. J Agric Food Sci 1992; 40:1531-5.

12. Manach C, Scalbert A, Morand C, Rémésy C, Jimenez L. Polyphenols: food sources and bioavailability. Am J Clin Nutr 2004; 79:727-47.

13. Parr AJ, Bolwell GP. Phenols in the plant and in man. The potential for possible nutritional enhancement of the diet by modifying the phenol content or profile. J Agric Food Chem 2000; 80:985-1012.

14. Sosulski FW, Krygier K, Hogge L. Importance of phenolic compounds for the characterization of fruit juices .J Agric Food Chem 1982; 30:337-40.

15. Price KR, Bacon JR, Rhodes MJC. Effect of storage and domestic processing on the content and composition of flavonol glucosides in onion (Allium cepa). J Agric Food Chem 1997; 45:938-42.

16. Crozier A, Lean MEJ, McDonald MS, Black C. Quantitative analysis of the flavonoid content of commercial tomatoes, onions, lettuce, and celery. J Agric Food Chem 1997; 45:590-5. including cancer, diabetes, cardio-vascular problems and aging. Although several biological effects based on epidemiological studies can be scientifically explained, the mechanism of action of some effects of polyphenols is not fully understood. A better knowledge of some variables of polyphenol bioavailability; such as the kinetics of absorption, accumulation and elimination, will facilitate the design of such studies. The role of polyphenols in human health is still a fertile area of research. Based on our current scientific understanding, polyphenols offer great hope for the prevention of chronic human diseases.

\section{Acknowledgements}

K.B.P. is a recipient of Senior Research Fellowship form Council of Scientific \& Industrial Research (CSIR), India.
17. D’Archivio M, Filesi C, Benedetto RD, Gargiulo R, Giovannini C, Masella R. Polyphenols, dietary sources and bioavailability. Ann Ist Super Sanità 2007; 43:348-61

18. Day AJ, Williamson G. Biomarkers for exposure to dietary flavonoids: a review of the current evidence for identification of quercetin glycosides in plasma. Br J Nutr 2001; 86:S105-10.

19. Setchell KD, Faughnan MS, Avades T, ZimmerNechemias L, Brown NM, et al. Comparing the pharmacokinetics of daidzein and genistein with the use of 13C-labeled tracers in premenopausal women. Am J Clin Nutr 2003; 77:411-9.

20. Duthie GG, Pedersen MW, Gardner PT, Morrice PC, Jenkinson AM, McPhail DB, Steele GM. The effect of whisky and wine consumption on total phenol content and antioxidant capacity of plasma from healthy volunteers. Eur J Clin Nutr 1998; 52:733-6.

21. Young JF, Nielsen SE, Haraldsdóttir J, Daneshvar B, Lauridsen ST, Knuthsen P, Crozier A, Sandström B, Dragsted LO. Effect of fruit juice intake on urinary quercetin excretion and biomarkers of antioxidative status. Am J Clin Nutr 1999; 69:87-94

22. Gee JM, DuPont MS, Rhodes MJ, Johnson IT. Quercetin glucosides interact with the intestinal glucose transport pathway. Free Radic Biol Med 1998; 25:19-25.

23. Crespy V, Morand C, Besson C, Manach C, Demigne C, Remesy C. Quercetin, but not its glycosides, is absorbed from the rat stomach. J Agric Food Chem 2002; 50:618-21.

24. Passamonti S, Vrhovsek U, Vanzo A, Mattivi F. Fast access of some grape pigments to the brain. J Agric Food Chem 2005; 53:7029-34.

25. Halliwell B, Zhao K, Whiteman M. The gastrointestinal tract: a major site of antioxidant action? Free Radic Res 2000; 33:819-30

26. Clifford MN. Chlorogenic acids and other cinnamates. Nature, occurence, dietary burden, absorption and metabolism. J Sci Food Agric 2000; 80:103343.

27. Olthof MR, Hollman PC, Katan MB. Chlorogenic acid and caffeic acid are absorbed in humans. J Nutr 2001; 131:66-71.

28. Kuhnau J. The flavonoids. A class of semi-essential food components: their role in human nutrition. World Rev Nutr Diet 1976; 24:117-91.

29. Lee MJ, Maliakal P, Chen L, Meng X, Bondoc FY, et al. Pharmacokinetics of tea catechins after ingestion of green tea and (-)-epigallocatechin-3-gallate by humans: formation of different metabolites and individual variability. Cancer Epidemiol Biomarkers Prev 2002; 11:1025-32.

30. Falany CN. Enzymology of human cytosolic sulfotransferases.Faseb J 1997; 11:206-16.
31. Spencer JP, Chowrimootoo G, Choudhury R, Debnam ES, Srai SK, Rice-Evans C.The small intestine can both absorb and glucuronidate luminal flavonoids. FEBS Lett 1999; 458:224-30.

32. Hollman PC, Tijburg LB, Yang CS. Bioavailability of flavonoids from tea. Crit Rev Food Sci Nutr 1997; 37:719-38.

33. Dangles O, Dufour C, Manach C, Morand C, Remesy C. Binding of flavonoids to plasma proteins. Methods Enzymol 2001; 335:319-33.

34. Dufour C, Loonis M, Dangles O. Inhibition of the peroxidation of linoleic acid by the flavonoid quercetin within their complex with human serum albumin. Free Radic Biol Med 2007; 43:241-52.

35. Vitrac X, Moni JP, Vercauteren J, Deffieux G Mérillon JM. Direct liquid chromatography analysis of resveratrol derivatives and flavanonols in wines with absorbance and fluorescence detection. Anal Chim Acta 2002; 458:103-10.

36. Luqman S, Rizvi SI. Protection of lipid peroxidation and carbonyl formation in proteins by capsaicin in human erythrocytes subjected to oxidative stress. Phytother Res 2006 20:303-6.

37. Pandey KB, Mishra N, Rizvi SI. Protective role of myricetin on markers of oxidative stress in human erythrocytes subjected to oxidative stress. Nat Prod Commun 2009; 4:221-6.

38. Pandey KB, Rizvi SI. Protective effect of resveratrol on markers of oxidative stress in human erythrocytes subjected to in vitro oxidative insult. Phytother Res 2009; In press.

39. Renaud S, de Lorgeril M. Wine, alcohol, platelets, and the French paradox for coronary heart disease. Lancet 1992; 339:1523-6.

40. Dubick MA, Omaye ST. Evidence for grape, wine and tea polyphenols as modulators of atherosclerosis and ischemic heart disease in humans. J Nutraceut Functional \& Med Foods 2001; 3:67-93.

41. Nardini M, Natella F, Scaccini C. Role of dietary polyphenols in platelet aggregation. A review of the supplementation studies. Platelets 2007; 18: 224-43.

42. Vita JA. Polyphenols and cardiovascular disease: effects on endothelial and platelet function. Am J Clin Nutr 2005; 81:292-7.

43. Aviram M, Dornfeld L, Rosenblat M, Volkova N, Kaplan M, Coleman R, Hayek T, Presser D, Fuhrman B. Pomegranate juice consumption reduces oxidative stress, atherogenic modifications to LDL, and platele aggregation: Studies in humans and in atherosclerotic apolipoprotein E-deficient mice. Am J Clin Nutr 2000; 71:1062-76.

44. García-Lafuente A, Guillamón E, Villares A, Rostagno MA, Martínez JA. Flavonoids as antiinflammatory agents: implications in cancer and cardiovascular disease. Inflamm Res 2009 ;58:537-52. 
45. Maeda K, Kuzuya M, Cheng XW, Asai T, Kanda S, Tamaya-Mori N, Sasaki T, Shibata T, Iguchi A. Green tea catechins inhibit the cultured smooth muscle cell invasion through the basement barrier. Atherosclerosis 2003; 166:23-30.

46. Demrow HS, Slane PR, Folts JD. Administration of wine and grape juice inhibits in vivo platelet activity and thrombosis in stenosed canine coronary arteries. Circulation 1995; 91:1182-8.

47. Schachinger V, Britten MB, Zeiher AM. Prognostic impact of coronary vasodilator dysfunction on adverse long-term outcome of coronary heart disease. Circulation 2002; 101:1899-906.

48. Duffy SJ, Keaney JF, Jr. Holbrook M, Gokce N, Swerdloff PL, Frei B, Vita JA. Short- and long-term black tea consumption reverses endothelial dysfunction in patients with coronary artery disease. Circulation 2001; 104:151-6.

49. Pirola L, Frojdo S. Resveratrol: One Molecule, Many Targets. IUBMB Life 2008; 60: 323-32.

50. Harikumar KB, Aggarwal BB. Resveratrol: a multitargeted agent for age-associated chronic diseases. Cell Cycle 2008; 7: 1020-35.

51. Cucciolla V, Borriello A, Oliva A, Galletti P, Zappia V, Della Ragione F. Resveratrol: from basic science to the clinic. Cell Cycle 2007; 6:2495-510.

52. Shakibaei M, Harikumar KB, Aggarwal BB. Resveratrol addiction: to die or not to die.Mol Nutr Food Res 2009; 53:115-28.

53. Peters U, Poole C, Arab L. Does tea affect cardiovascular disease? A meta-analysis. Am J Epidemiol 2001; 154:495-503.

54. Yang CS, Landau JM, Huang MT, Newmark HL. Inhibition of carcinogenesis by dietary polyphenolic compounds. Ann Rev Nutr 2001; 21:381-406.

55. Johnson IT, Williamson G, Musk SRR. Anticarcinogenic factors in plant foods: A new class of nutrients? Nutr Res Rev 1994; 7:175-204.

56. Talalay P, De Long MJ, Prochaska HJ. Identification of a common chemical signal regulating the induction of enzymes that protect against chemical carcinogenesis. Proc Natl Acad Sci USA 1988; 85:82615 .

57. Khan N, Mukhtar H. Multitargeted therapy of cancer by green tea polyphenols. Cancer Lett 2008; 269:269-80.

58. Sharma V, Rao LJ.A thought on the biological activities of black tea. Crit Rev Food Sci Nutr 2009; 49:379-404.

59. Kamaraj S, Vinodhkumar R, Anandakumar P, Jagan $S$, Ramakrishnan G, Devaki T. The effects of quercetin on antioxidant status and tumor markers in the lung and serum of mice treated with benzo(a)pyrene. Biol Pharm Bull 2007; 30:2268-73.

60. Athar M, Back JH, Tang X, Kim KH, Kopelovich L, Bickers DR, Kim AL. Resveratrol: a review of preclinical studies for human cancer prevention. Toxicol Appl Pharmacol 2007; 224:274-83.

61. Rizvi SI, Zaid MA. Intracellular reduced glutathione content in normal and type 2 diabetic erythrocytes: effect of Insulin and (-)epicatechin. J Physiol Pharmacol 2001; 52:483-8.

62. Rizvi SI, Zaid MA. Impairment of sodium pump and $\mathrm{Na} / \mathrm{H}$ exchanger in erythrocytes from non-insulin dependent diabetes mellitus patients: effect of tea catechins. Clin Chim Acta 2005; 354:59-67.
63. Rizvi SI, Zaid MA, Anis R, Mishra N. Protective role of tea catechins against oxidation-induced damage of type 2 diabetic erythrocytes. Clin Exp Pharmacol Physiol 2005; 32:70-5.

64. Rizvi S I, Zaid M A. Insulin like effect of epicatechin on membrane acetylcholinesterase activity in type 2 diabetes mellitus. Clin Exp Pharmacol Physiol 2001; 28:776-8

65. Matsui T, Ebuchi S, Kobayashi M, Fukui K, Sugita K, Terahara N, Matsumoto K. Anti-hyperglycemic effect of diacylated anthocyanin derived from Ipomoea batatas cultivar Ayamurasaki can be achieved through the alpha-glucosidase inhibitory action. J Agric Food Chem 2002; 50:7244-8.

66. Matsui T, Ueda T, Oki T, Sugita K, Terahara N, Matsumoto K. Alpha-Glucosidase inhibitory action of natural acylated anthocyanins. 2. alpha-Glucosidase inhibition by isolated acylated anthocyanins.] Agric Food Chem 2001; 49:1952-6.

67. Dembinska-Kiec A, Mykkänen O, Kiec-Wilk B Mykkänen H. Antioxidant phytochemicals against type 2 diabetes.Br J Nutr 2008; 99:109-17.

68. Milne JC, Lambert PD, Schenk S, Carney DP, Smith JJ, Gagne DJ, et al. Small molecule activators of SIRT1 as therapeutics for the treatment of type 2 diabetes. Nature 2007; 450:712-6.

69. Chen WP, Chi T C, Chuang LM, Su MJ. Resveratrol enhances insulin secretion by blocking K(ATP) and K(V) channels of beta cells. Eur J Pharmacol 2007; 568:269-77.

70. Rizvi SI, Mishra M. Anti-oxidant effect of quercetin on type 2 diabetic erythrocytes. J Food Biochem 2009; 33:404-15

71. Lee WC, Wang CJ, Chen YH, Hsu JD, Cheng SY, Chen HC, et al. Polyphenol extracts from Hibiscus sabdariffa Linnaeus attenuate nephropathy in experimental type 1 diabetes. J Agric Food Chem 2009; 57:2206-10.

72. Barone E, Calabrese V, Mancuso C. Ferulic acid and its therapeutic potential as a hormetin for age-related diseases. Biogerontology 2009; 10:97-108.

73. Jung EH, Kim SR, Hwang IK, Ha TY. Hypoglycemic effects of a phenolic acid fraction of rice bran and ferulic acid in C57BL/KsJ-db/db mice. J Agric Food Chem 2007; 55:9800-4.

74. Harman D. Free radical theory of aging: an update. Ann N Y Acad Sci 2006; 1067:1-12.

75. Rizvi SI, Maurya PK. Alterations in antioxidant enzymes during aging in humans. Mol Biotechnol 2007; 37:58-61.

76. Rizvi SI, Maurya PK. Markers of oxidative stress in erythrocytes during aging in human. Ann N Y Acad Sci 2007; 1100:373-82.

77. Cao G, Booth S L, Sadowsky J A, Prior R L. Increases in human plasma antioxidant capacity after consumption of controlled diets high in fruit and vegetables. Am J Clin Nutr 1998; 68:1081-7.

78. Joseph JA, Shukitt-Hale B, Casadesus G. Reversing the deleterious effects of aging on neuronal communication and behavior: beneficial properties of fruit polyphenolic compounds. Am J Clin Nutr 2005; 81:313-6.

79. Seeram NP, Cichewicz R H, Chandra A, Nair MG. Cyclooxygenase inhibitory and antioxidant compounds from crabapple fruits. J Agric Food Chem 2003; 51:1948-51.
80. Shukitt-Hale B, Lau FC, Joseph JA. Berry Fruit Supplementation and the Aging Brain. J Agric Food Chem 2008; 56:636-41.

81. Maurya PK, Rizvi SI. Protective role of tea catechins on erythrocytes subjected to oxidative stress during human aging. Nat Prod Res 2008; 1-8.

82. Markus MA, Morris BJ. Resveratrol in prevention and treatment of common clinical conditions of aging. Clin Interv Aging 2008; 3:331-9.

83. Barger JL, Kayo T, Vann JM, Arias EB, Wang J, Hacker TA, et al. A low dose of dietary resveratrol partially mimics caloric restriction and retards aging parameters in mice. PLoS One 2008; 3; 22264.

84. Belinha I, Amorim MA, Rodrigues P, de Freitas V, Moradas-Ferreira P, Mateus N, et al. Quercetin increases oxidative stress resistance and longevity in Saccharomyces cerevisiae. J Agric Food Chem 2007; 55:2446-51.

85. Letenneur L, Proust-Lima C, Le Gouge A, Dartigues J, Barberger-Gateau P. Flavonoid intake and cognitive decline over a 10-year period. Am J Epidemiol 2007; 165:1364-71.

86. Scarmeas N, Luchsinger J A, Mayeux R, Stern Y. Mediterranean diet and Alzheimer disease mortality. Neurology 2007; 69: 1084-93.

87. Dai Q, Borenstein AR, Wu Y, Jackson JC, Larson EB. Fruit and vegetable juices and Alzheimer's disease: the Kame Project. Am J Med 2006; 119: 751-9.

88. Singh M, Arseneault M, Sanderson T, Murthy V, Ramassamy C. Challenges for research on polyphenols from foods in Alzheimer's disease: Bioavailability, metabolism, and cellular and molecular mechanisms. J Agric Food Chem 2008; 56: 4855-73.

89. Aquilano K, Baldelli S, Rotilio G, Ciriolo MR. Role of nitric oxide synthases in Parkinson's disease: a review on the antioxidant and anti-inflammatory activity of polyphenols. Neurochem Res 2008; 33:2416-26.

90. Rossi L, Mazzitelli S, Arciello M, Capo CR, Rotilio G. Benefits from dietary polyphenols for brain aging and Alzheimer's disease. Neurochem Res 2008; 33:2390-400.

91. Tabak C, Arts ICW, Smit HA, Heederik D, Kromhout D. Chronic obstructive pulmonary disease and intake of catechins, flavonols, and flavones. The MORGEN study. Am J Respir Crit Care Med 2001; 164:61-4.

92. Woods RK, Raven JM, Wolfe R, Ireland PD, Thien FCK, Abramson MJ, Food and nutrient intakes and asthma risk in young adults. Am J Clin Nutr 2003; 78:414-21

93. Smith LJ, Holbrook JT, Wise R, Blumenthal M, Dozor AJ, Mastronarde J. et al. Dietary intake of soy genistein is associated with lung function in patients with asthma. J Asthma 2004; 41: 833-43.

94. Nakajima D, Kim CS, Oh TW, Yang CY, Naka T, Igawa $S$, Ohta F. Suppressive effects of genistein dosage and resistance exercise on bone loss in ovariectomized rats. J Physiol Anthropol Appl Human Sci 2001; 20:285-91.

95. Kim J, Hwang JS, Cho YK, Han Y, Jeon YJ, Yang KH. Protective effects of (-)-epigallocatechin-3gallate on UVAand UVB-induced skin damage. Skin Pharmacol Appl Skin Physiol 2001; 14:11-9. 


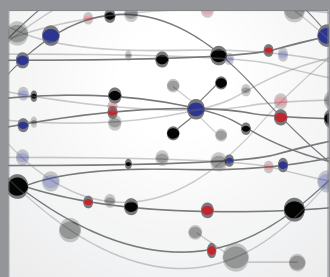

The Scientific World Journal
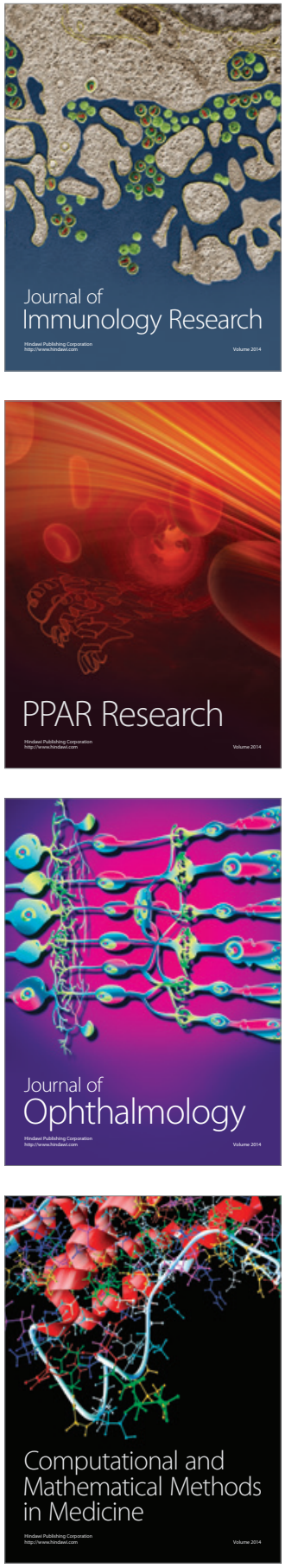

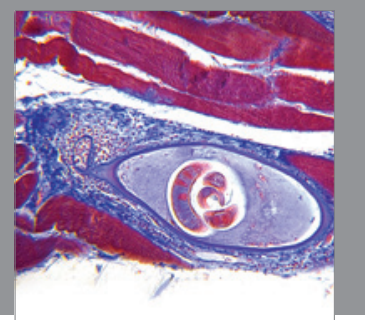

Gastroenterology

Research and Practice
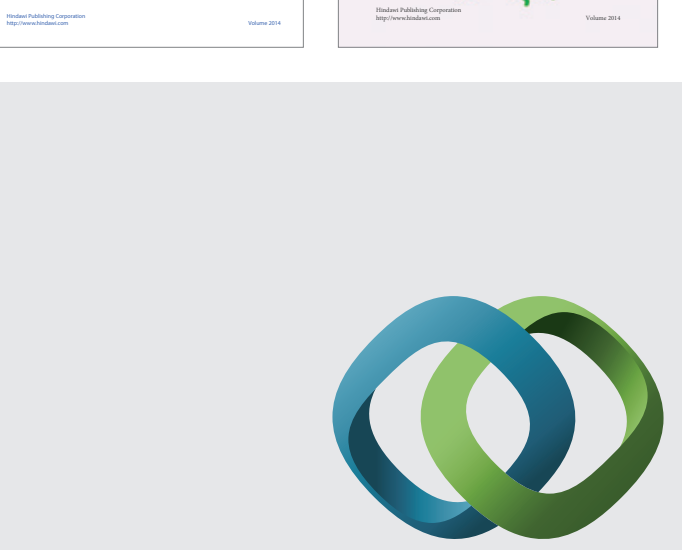

\section{Hindawi}

Submit your manuscripts at

http://www.hindawi.com
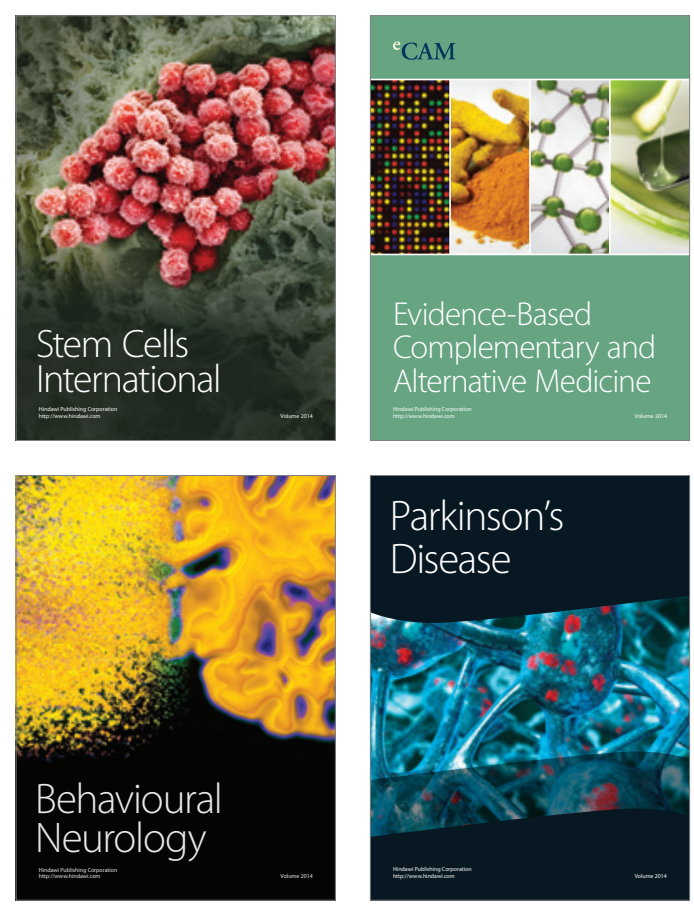

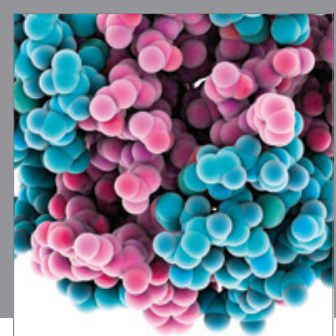

Journal of
Diabetes Research

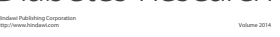

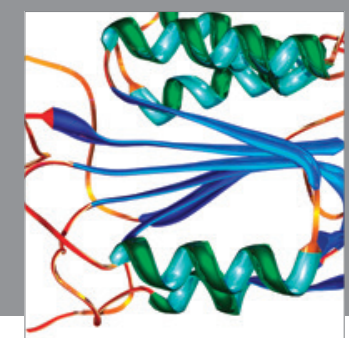

Disease Markers
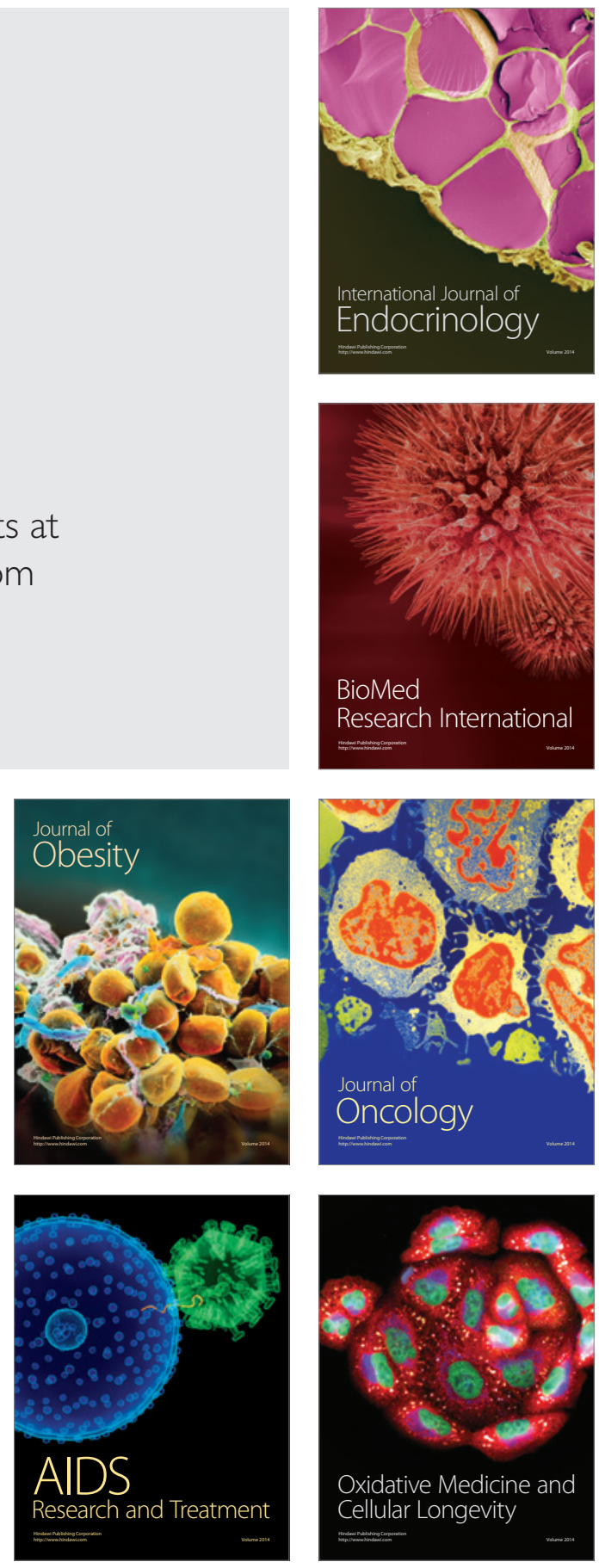